\title{
A rare case of fibrosarcoma of ovary
}

\author{
Humaeel Ali ${ }^{1, *}$, M. B. Swami ${ }^{2}$ \\ ${ }^{1}$ Junior Resident, ${ }^{2}$ Professor, Dept. of Obstetrics and Gynaecology, R. D. Gardi Medical College, Ujjain, Madhya Pradesh, India
}

*Corresponding Author:

Email: humaredd@gmail.com

\begin{abstract}
Ovarian fibroma comprises $3 \%$ of all ovarian neoplasms and their malignant counterpart is very rare. Till today only 100 cases have been reported in the literature. ${ }^{1}$ Woman aged 60 years, postmenopausal, reported with pain in abdomen from 3 years. A $10 \times 6 \mathrm{~cm}$ mobile, firm ovarian mass was felt through left fornix with no other significant findings was noted in general and systemic examination. All tumor markers were within normal limit. USG detected left ovarian mass. Laparotomy showed white, firm, solid mass arising from left ovary of $10 x 7 \mathrm{~cm}$ size. No evidence of metastases. Histopathology examination revealed ovarian fibrosarcoma.
\end{abstract}

Keywords: Fibrosarcoma ovary, Ovarian malignancy.

\section{Introduction}

Sex cord stromal tumors of the fibroma thecoma group account for $9 \%$ of ovarian tumors. ${ }^{2}$ Ovarian fibroma comprises $3 \%$ of ovarian neoplasms and their malignant counterpart even less common. Amongst this category of tumors a further division is done ranging from benign fibromas, mitotically active fibromas and fibrosarcomas. Primary ovarian fibrosarcomas are extremely rare entities associated with hemorrhage and necrosis and presence of large veins on surface. ${ }^{1}$ This case report is being presented owing to its rarity in gynecological neoplasms.

\section{Case Summary}

A 60 year old postmenopausal woman presented with complaint of dull aching pain in lower abdomen for $2-3$ years on $10^{\text {th }}$ October 2016. She attained menopause 10 years back. Obstetric history was para3 abortion 0 living 3. On examination her vitals were normal, pallor absent. Cardiovascular and Respiratory system showed normal findings. Per abdominal examination showed mass arising from pelvis extending above pubic symphysis and was non tender, mobile with no ascites, liver spleen were not palpable. Per vaginal examination showed a $10 \times 6 \mathrm{~cm}$ left ovarian mass which was mobile, firm, non tender with no deposits in pouch of Douglas. All ovarian tumor markers were within normal limit. Ultrasound of pelvis showed $10 \times 6 \mathrm{~cm}$ left ovarian mass; $X$ ray chest was normal. Laparatomy was done on $15^{\text {th }}$ October 2016. Firm, solid, white left ovarian tumor of size $10 \times 6 \mathrm{~cm}$ size was detected. Surgical staging was done. No deposits were identified except on the omentum. Hysterectomy with right salpingo opherectomy and Left ovarectomy was done and sent for histopathology. The lesion on section showed early changes of fibrosarcoma. Cut section of cervix revealed benign squamous hyperplasia with chronic cervicitis and endometrium was proliferative non secretory with atrophic glands.

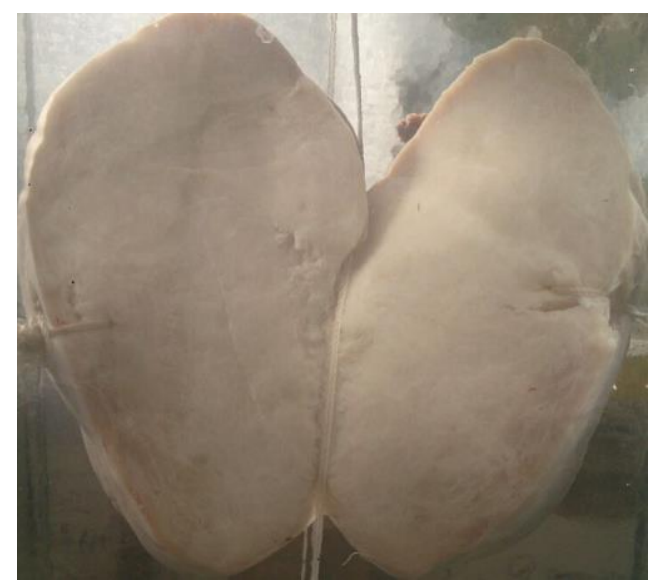

Fig. 1: Gross appearance with cut section showing homogenous solid white mass 


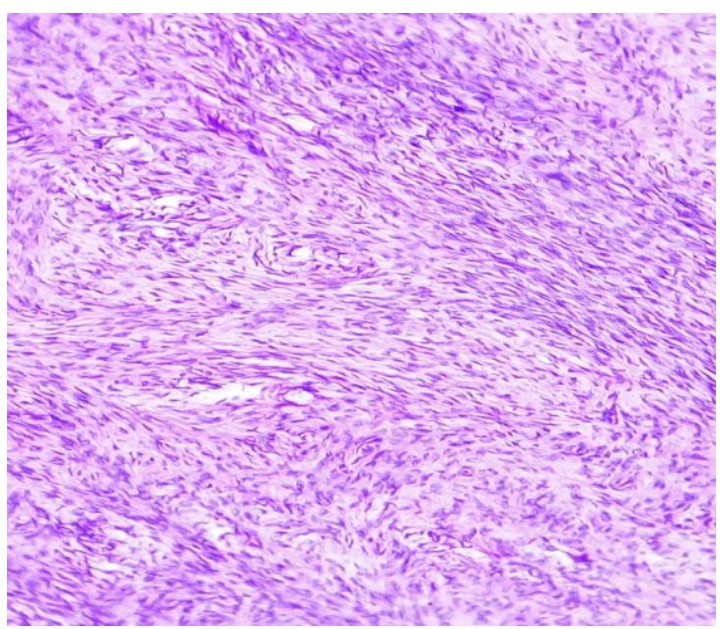

Fig. 2: H \& E stained section showing spindle shaped cells arranged in storiform pattern (Low power view 40 X)

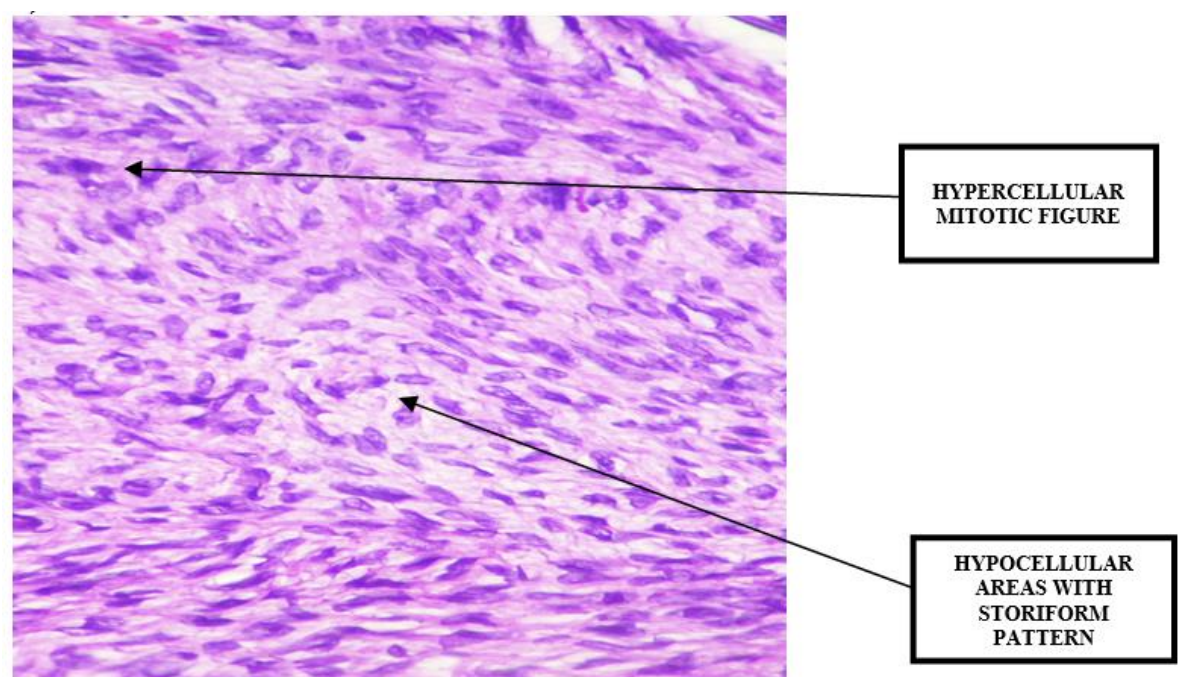

Fig. 3: H \& E section showing hypercellular areas with mitotic figures (High power view 100X)

\section{Discussion}

Primary ovarian fibrosarcoma is a rare entity. ${ }^{3}$ Isolated cases have been noted of unilateral primary fibrosarcoma. ${ }^{4,5}$ These tumors may either arise de novo or may undergo sarcomatous change in a pre existing fibroma of ovary. There is no age predilection but are usually seen amongst perimenopausal and menopausal women. They may attain very large sizes and may have hemorrhage and necrosis in tissue substance. The most significant feature to identify these tumors is the microscopic and immunohistochemical staining properties. In 1981, Pratt and Scully found mitotic activity to be the most important factor in diagnosing fibrosarcoma of ovary. ${ }^{6}$ They suggested that a tumor having $<3 / 10$ high power field mitotic figures be considered as mitotically active fibromas and those with $>4 / 10 \mathrm{hpf}$ be diagnosed as fibrosarcomas. ${ }^{6}$ Usually fibrosarcomas are associated with cellular atypia and complications as hemorrhage and necrosis and adhesions with other visceral structures. Irving et al studied 75 cases of ovarian fibrosarcomas, of which 45 were mitotically active fibromas. They concluded that not all histological differences can be discriminatory between a fibrosarcoma and mitotically active fibroma. ${ }^{7}$ In our case histopathology revealed cellular atypia and mitotic figures $>4 / 10$ HPF suggestive of fibrosarcomatous change.

Poor prognostic factors of fibrosarcoma include high grade, high cellularity with minimal collagen, mitotic rates $>20 / 10$ hpf, necrosis and little collagen. ${ }^{8}$ Many of these are not aggressive in nature, however a strong correlation exists between the histological grade, nuclear atypia and positivity for immunohistochemical staining for $\mathrm{Ki}-67$ antigen. Immunohistochemical staining for Ki-67 antigen is used to assess the proliferative activity of various tumors and the Ki-67 labeling index is reported to be related to other prognostic factors in case of soft tissue sarcoma. ${ }^{9}$

Ovarian tumor markers play little role in confirmation of fibrosarcomas. In our case all ovarian tumor markers were within normal limits. Similar studies conducted by Shoji Kaku et $\mathrm{al}^{10}$ and Biplab K 
Biswas et al $^{11}$ showed ovarian tumor markers in normal range.

Fibrosarcomas can attain very large sizes. The tumor size in our case was $10 \times 6 \mathrm{~cm}$ whereas in the case report by Shoji et $\mathrm{al}^{10}$ and Chandra Mathur et $\mathrm{al}^{8}$ it was $6 \times 6 \mathrm{~cm}$ and 20x20 $\mathrm{cm}$ respectively.

Despite the reliability of mitotic counts as differentiating feature between mitotically active fibromas and fibrosarcomas; it is not the only criteria helpful in diagnosing either variety and differentiating from other spindle cell lesions. Tsuji et al [9] established that the MIB -1 (Ki-67) labeling index (LI) in atypical fibromatous lesions of the ovary was reflective of the potential aggressive nature of tumor.

\section{Conclusion}

Sarcomatous change in ovarian fibroma is an extremely rare entity. These tumors should be differentiated from their benign counterparts. Surgical removal remains mainstay of treatment. Our patient successfully underwent surgical staging and tumor resection and is free from recurrent growth even in absence of postoperative chemotherapy.

\section{References}

1. Garcia Jimenez A, Castellvi J, Perez Benavente A, Diaz de Corcuera Frutos I, Ramon Y, Cajal S: Ovarian Fibrosarcoma: Clinicopathologic Considerations about the Intraoperative and Post-Surgical Procedures. Case Report Med. 2009, 2009:802817.

2. Charles J. Zaloudek, Karuna Garg. Chapter 13, Tumors of the Female Genital Tract. In Diagnostic Histopathology Of Tumors, Christopher D.M. Fletcher. $4^{\text {th }}$ edi. Elsevier limited, Philadelphia; 2013. Pp.658-730.

3. Azoury RS, Woodruff JD: Primary ovarian sarcomas, report of 43 cases from the Emil Novak Ovarian Tumor Registry. Obstetrics and Gynecology. 1971,37(6):920-41.

4. Stellato G, Bonito MD, Tramontana S. Primary fibrosarcoma of the ovary. Acta obstetricia et gynecologica Scandinavica. 1995 Jan 1;74(8):649-52.

5. Ikegami A, Ono I, Harada T, Ogura Y, Sumori K, Kouno K, Uchida S. A case of fibrosarcoma of the ovary. Nihon Sanka Fujinka Gakkai Zasshi. 1996 Dec;48(12):1177-9.

6. Prat J, Scully RE: Cellular fibromas and fibrosarcomas of the ovary: a comparative clinicopathologic analysis of seventeen cases. Cancer. 1981,47:2663-70.

7. Irving JA, Alkushi A, Young RH, Clement PB: Cellular fibromas of the ovary: a study of 75 cases including 40 mitotically active tumors emphasizing their distinction from fibrosarcoma. American Journal of Surgical Pathology. 2006,30(8):929-38.

8. Mathur C, Agarwal M, Kothari DC, Tailor SB. Primary Ovarian Fibrosarcoma-A Rare Case Report. IOSR Journal of Dental and Medical Sciences (IOSRJDMS).;1(15):65-8.

9. Tsuji T, Kawauchi S, Utsunomiya T, Nagata Y, Tsuneyoshi M: Fibrosarcoma versus cellular fibroma of the ovary: a comparative study of their proliferative activity and chromosome aberrations using MIB-1 immunostaining, DNA flow cytometry, and fluorescence in situ hybridization. Am J Surg Pathol. 1997, 21:52-9.

10. S. Kaku, N. Takeshima, F. Akiyama, R. Furuta, Y. Hirai, and K.Takizawa, "A unique fibrous tumor of the ovary: fibrosarcoma or mitotically active cellular fibroma?" Anticancer Research, vol. 27, no. 6C, pp.4365-9,2007.

11. Ray S, Biswas BK, Mukhopadhyay S. Giant primary ovarian fibrosarcoma: Case report and review of pitfalls. Journal of cytology. 2012 Oct 1;29(4):255. 